Draft VERSION MAY 28, 2018

Preprint typeset using $\mathrm{LAT}_{\mathrm{E} X}$ style emulateapj v. 14/09/00

\title{
COSMOLOGICAL BLACK HOLE SPIN EVOLUTION BY MERGERS AND ACCRETION
}

\author{
Emanuele Berti ${ }^{1}$ And Marta Volonteri ${ }^{2}$ \\ Draft version May 28, 2018
}

\begin{abstract}
Using recent results from numerical relativity simulations of black hole mergers, we revisit previous studies of cosmological black hole spin evolution. We show that mergers are very unlikely to yield large spins, unless alignment of the spins of the merging holes with the orbital angular momentum is very efficient. We analyze the spin evolution in three specific scenarios: (1) spin evolves only through mergers, (2) spin evolves through mergers and prolonged accretion episodes, (3) spin evolves through mergers and short-lived (chaotic) accretion episodes. We study how different diagnostics can distinguish between these evolutionary scenarios, assessing the discriminating power of gravitational-wave measurements and X-ray spectroscopy. Gravitational radiation can produce three different types of spin measurements, yielding respectively the spins of the two black holes in a binary inspiral prior to merger, the spin of the merger remnant (as encoded in the ringdown waves), and the spin of "single" black holes during the extreme mass-ratio inspiral (EMRI) of compact objects. The latter spin population is also accessible to iron-line measurements. We compute and compare the spin distributions relevant for these different observations. If iron-line measurements and gravitational-wave observations of EMRIs only yield dimensionless spins $j=J / M^{2}>0.9$, then prolonged accretion should be responsible for spin-up, and chaotic accretion scenarios would be very unlikely. If only a fraction of the whole population of low-redshift black holes spins rapidly, spin-alignment during binary mergers (rather than prolonged accretion) could be responsible for spin-ups.
\end{abstract}

Subject headings: cosmology: theory - black holes - gravitational waves - galaxies: evolution

\section{INTRODUCTION}

In general relativity, the properties and dynamics of astrophysical black holes (BHs) are determined only by their masses and spins. There is observational evidence that BHs with masses in the range $\sim 10^{6}-10^{9} M_{\odot}$ exist in the bulges of nearly all local, massive galaxies, including our own. BH mass measurements show an almost-linear relation between the $\mathrm{BH}$ mass and the mass of the galactic bulge hosting the BH. Spin measurements are generally more uncertain (Narayan 2005; Miller 2007), and the cosmological coevolution of $\mathrm{BH}$ masses and spins is a significant open problem in our understanding of quasars and AGNs (Gammie et al. 2004; Shapiro 2005).

Massive BH formation scenarios (Volonteri et al. 2003) are important in the planning of the space-based gravitational-wave detector LISA. Cosmological BH evolution affects the rates of detectable events (Berti 2006; Sesana et al. 2007), data analysis strategies (Arnaud et al. 2007) and the science performance of the instrument, including its parameter estimation capabilities. $\mathrm{BH}$ spins have a strong impact on source modeling and parameter estimation, because they induce precession in the orbits of inspiralling $\mathrm{BH}$ binaries and affect the radiation emitted in the merger and ringdown phases.

Volonteri et al. (2005) (henceforth paper I) studied the distribution of massive $\mathrm{BH}$ spins and its evolution with cosmic time under the combined effect of mergers and accretion in the context of hierarchical galaxy formation theories. They found that gas accretion affects the spin evolution more than mergers, and that prolonged accretion efficiently spins holes up to $j \sim 1$.

1 Jet Propulsion Laboratory, California Institute of Technology, Pasadena, CA 91109, USA

2 University of Michigan, Astronomy Department, Ann Arbor, MI 48109, USA
Until recently, the spin of the $\mathrm{BH}$ resulting from generic BH mergers was estimated by heuristic arguments (see e.g. Merritt \& Ekers (2002)). Hughes \& Blandford (2003) (henceforth HB) estimated the merger remnant's spin using an extrapolation to comparable-mass binaries of results valid for small mass ratios, $q=M_{2} / M_{1} \ll 1$, showing that mergers typically spin BHs down. Their calculations become unreliable for "major" mergers with $q \sim 1$. Recent breakthroughs in numerical relativity have provided us with a quantitative understanding of the spins and recoil velocities resulting from comparable-mass binary $\mathrm{BH}$ mergers: see Pretorius (2007) for a review. Here we update the analysis of paper I, that used the HB model. For the first time, we implement numerical relativity results to compute the $\mathrm{BH}$ spin resulting from generic mergers in hierarchical models of $\mathrm{BH}$ formation. We study how the efficiency of spin alignment with the orbital angular momentum L affects upper and lower limits on the remnant's spin by considering three different scenarios: (1) complete isotropy, (2) efficient alignment of the spin of the more massive hole with L, so that the smaller hole orbits in the equatorial plane of the larger, and (3) alignment of both spins with L.

Spin-up is a natural consequence of prolonged disc-mode accretion: any hole that has doubled its mass by capturing material with constant angular momentum axis would end up spinning rapidly (Bardeen 1970; Thorne 1974). However, when the angular momentum of the accretion disc is misaligned with respect to the direction of the $\mathrm{BH}$ spin, accretion of counter-rotating material can cause a spin-down of the hole. The orbits in the inner accretion disc co-rotate 
or counter-rotate depending on the ratio between the angular momentum of the disc and of the hole: if the cosine of the inclination angle $\cos \phi<-j_{d} /(2 j)$, where $j_{d}$ is the total angular momentum of the accretion disc, then the disc counter-rotates (King et al. 2005). Sustained accretion from a counter-rotating disc spins down a maximallyrotating hole to $j=0$ after the hole has increased in mass by a factor $\sim \sqrt{3 / 2}$ (Bardeen 1970). A complete overflip eventually occurs, and then accretion of co-rotating material acts to spin up the $\mathrm{BH}$ again: a $180^{\circ}$ flip of the spin of an extreme-Kerr hole will occur after the $\mathrm{BH}$ triples in mass. Paper I argued that the lifetime of quasars is long enough that the innermost regions of accretion discs align with BH spins (possibly through spin flips), and hence all AGN BHs should have large spins.

The picture of spin evolution via prolonged accretion was questioned by King et al. (2005). King \& Pringle (2006) suggest that accretion always proceeds via very small (and short) episodes, caused by fragmentation of the accretion disc where it becomes self-gravitating. The hole would then accrete each time an amount of mass corresponding to a tiny fraction of its own mass, and all episodes would have uncorrelated directions ("chaotic accretion"). Since counter-rotating material spins BHs down more efficiently than co-rotating material spins them up, this scenario implies that $\mathrm{BH}$ spins are very small: accretion of randomly oriented droplets of gas would rapidly spin down any BH that had its spin increased by a "major" merger. The only rapidly spinning holes would then be those which have recently experienced a merger, and have not accreted any matter afterwards. King et al. (2008) refined their chaotic accretion scenario by statistical considerations, concluding that accretion very rapidly adjusts the hole's spin parameter to average values $j \sim 0.1-0.3$ from any initial conditions, but with significant fluctuations $\Delta j \sim \pm 0.2$ about these values. In this paper we compare the prolonged-accretion scenario and the chaotic accretion scenario, showing that rapidly spinning holes should be extremely rare if chaotic accretion is the norm.

In Section 2 we review numerical simulations of $\mathrm{BH}$ mergers and discuss their predictions for the final spin. In Section 3 we present our main results on cosmological spin evolution in different merger scenarios, with and without accretion.

\section{FINAL SPIN FROM BINARY MERGER SIMULATIONS}

In the last two years there has been enormous progress in the numerical simulation of $\mathrm{BH}$ binaries. We now know that equal-mass non-spinning binaries produce a rotating (Kerr) $\mathrm{BH}$ with final dimensionless spin parameter $\left|\mathrm{j}_{\text {fin }}\right| \simeq 0.69$. For unequal-mass non-spinning mergers with mass ratio $q$, the final spin is well fitted by the sum of two terms: $\left|\mathrm{j}_{\mathrm{fin}}\right| \simeq 2 \sqrt{3} q /(1+q)^{2}-2.029 q^{2} /(1+q)^{4}$. The first term is an extrapolation to comparable masses of the orbital angular momentum of a particle at the innermost stable circular orbit of a non-rotating $\mathrm{BH}$, and the second term accounts for the angular momentum radiated in the final plunge (Berti et al. 2007a).

A significant sample of spinning binary $\mathrm{BH}$ merger simulations is now available. Different groups showed that when the initial spins are large and aligned with the orbital angular momentum L the binary "hangs up", radiating more energy and angular momentum (Campanelli et al. 2006; Baker et al. 2007; Pollnev et al. 2007; Marronetti et al. 2007). Even accounting for the additional angular momentum due to orbital eccentricity, no violation of cosmic censorship should occur: a binary $\mathrm{BH}$ merger should always result in the formation of a Kerr BH (Sperhake et al. 2007). Simulations have also been carried out for some "generic" orientations of the initial spins, including configurations leading to spin-flips or to Schwarzschild remnants (Campanelli et al. 2007; Herrmann et al. 2007; Tichy \& Marronetti 2007; Berti et al. 2007b).

Unfortunately, covering the whole parameter space by numerical simulations is computationally costly and not practical. Semi-analytical models for the Kerr parameter of the final hole are particularly useful for astrophysical applications. One such model, based on point-particle analogies, has been proposed by Buonanno et al. (2007). Their model can reproduce the final spin computed by numerical simulations within a few percent, the disagreement being larger when both spins are large and anti-aligned with L. Here we use semi-analytical fitting formulas of numerical results derived by Rezzolla et al. (2007a,b, c). Unfortunately, due to the inaccuracy of large-spin simulations and to errors in the fitting parameters, the final spin $\left|j_{\text {fin }}\right|$ predicted by the fits can be slightly larger than the Kerr bound when the initial Kerr parameters of the holes $\left|\mathrm{j}_{i}\right|$ $(i=1,2)$ are large and aligned with $L$. To get rid of this undesired feature we simply introduce a cutoff on the final spin at $\left|j_{\text {fin }}\right|=1$. An elegant and potentially very accurate recipe to compute the final spins has been proposed by Boyle et al. (2007) using symmetry arguments. Some parameters in their "spin expansion" are presently undetermined, but in principle they can be fixed by a reasonably small number of dedicated numerical simulations.

The spin expansion model should be able to provide accurate and general predictions for the final spin in the near future, but the fitting formula of Rezzolla et al. (2007a) is accurate enough for our preliminary exploration. Their formula can be used to compute $\left|\mathrm{j}_{\text {fin }}\right|\left(q,\left|\mathrm{j}_{1}\right|,\left|\mathrm{j}_{2}\right|, \cos \alpha, \cos \beta, \cos \gamma\right)$, where $\cos \alpha=\hat{\mathrm{j}}_{1} \cdot \hat{\mathrm{j}}_{2}$, $\cos \beta=\hat{\mathrm{j}}_{1} \cdot \hat{\mathrm{L}}$ and $\cos \gamma=\hat{\mathrm{j}}_{2} \cdot \hat{\mathrm{L}}$.

As a starting point, for comparison with $\mathrm{HB}$, we computed the final spin resulting from a merger where the smaller BH is non-spinning $\left(\left|\mathrm{j}_{2}\right|=0\right)$. Since $\left|\mathrm{j}_{2}\right|=0$ the angles $\cos \alpha$ and $\cos \gamma$ are irrelevant, and only $\cos \beta$, the orbital inclination of the smaller hole, matters. Contour plots of the final spin $\left|j_{\text {fin }}\right|$ in the $\left(\cos \beta,\left|j_{1}\right|\right)$ plane, as obtained from the fitting formula, are shown in our Figure 1 . that should be compared with Figure 1 in HB (notice that our angle $\beta$ is denoted by $\iota$ in their work). For $q=1 / 10$ our calculations are in nice agreement with HB: this is a useful consistency check of our approach in the small massratio limit. However, observational and theoretical arguments suggest that the coalescence of comparable-mass BHs should be rather common (Bogdanovic et al. 2007). When $q \sim 1$ and $\left|\mathrm{j}_{2}\right|=0$, we find that the range of variability of the final spin is sensibly smaller than predicted by HB: $0.3 \lesssim\left|\mathrm{j}_{\text {fin }}\right| \lesssim 0.9$ for $q=1 / 2$, and $0.5 \lesssim\left|\mathrm{j}_{\text {fin }}\right| \lesssim 0.8$ for $q=1$.

In Figure 2 we drop the assumption $\left|j_{2}\right|=0$ and we show contour plots of $\left|j_{\text {fin }}\right|$ in the $\left(\left|j_{1}\right|,\left|j_{2}\right|\right)$ plane, for se- 
lected values of $q$. To deal with the angular dependence we consider three different merger scenarios:

(1) Isotropic mergers: for each pair of initial spin magnitudes $\left(\left|\mathrm{j}_{1}\right|,\left|\mathrm{j}_{2}\right|\right)$ we average over $(\cos \alpha, \cos \beta, \cos \gamma)$, assuming isotropy on all three angles. This situation should be common in "dry" mergers, i.e. when holes do not accrete during merger, evolving solely via stellar dynamical processes. In vacuum, post-Newtonian equations predict that spin-orbit resonances will produce alignment of the spins in a very small region of parameter space (Schnittman 2004; Bogdanović et al. 2007), so isotropy should be a good assumption in the absence of accretion discs or gas. For all values of $q$, the minimum final spin results from non-spinning mergers with $\left|\mathrm{j}_{1}\right|=\left|\mathrm{j}_{2}\right|=0$. Naively we could expect that the average over all angles $(\cos \alpha, \cos \beta, \cos \gamma)$ should produce a final spin that is very close to the spin produced by non-spinning mergers, at least for equal masses. As we consider larger mass ratios the larger $\mathrm{BH}$ should play a more important role, so (on average) the final spin should be slightly larger than the value predicted by non-spinning mergers. This expectation is confirmed by the top row of Figure 2. In general, our calculations are in agreement with the HB conclusion that isotropic mergers tend to "spin-down" a fast-spinning hole.

(2) (Anti)aligned mergers: $\cos \alpha= \pm \cos \beta= \pm \cos \gamma=$ 1 , so the BH spins are always parallel (aligned or antialigned) with L. In the "wet merger" scenario proposed by Bogdanović et al. (2007), alignment (upper signs in \pm ) should be more likely than antialignment (lower signs), because the cumulative angular momentum of the accretion disc is much larger than the angular momentum of the BHs (but see King et al. 2005; Lodato \& Pringle 2006). If alignment is indeed the norm, we should focus on the top right quadrant of the contour plots in the central row. Then the minimum spin is still obtained when $\left|\mathrm{j}_{1}\right|=\left|\mathrm{j}_{2}\right|=0$, as in the isotropic case. The main difference is that now the maximum spin can be quite close to one, with a lower limit $\left|\mathrm{j}_{\text {fin }}\right| \sim 0.96$ for equal-mass mergers.

(3) Equatorial mergers: this case is intermediate between cases (1) and (2). Here the smaller $\mathrm{BH}$ inspirals in the equatorial plane of the larger $(\cos \beta=1)$, but for each pair $\left(\left|\mathrm{j}_{1}\right|,\left|\mathrm{j}_{2}\right|\right)$ we average assuming an isotropic distribution in $(\cos \alpha, \cos \gamma)$. Equatorial mergers could occur if Newtonian dynamical friction in a flattened system brings the smaller hole into the plane of a central (gaseous or stellar) disc before it gets into the relativistic regime. All mergers with $q \geq 1 / 2$ produce an average final spin $\left|\mathrm{j}_{\mathrm{fin}}\right| \leq 0.92$, and $\left|\mathrm{j}_{\text {fin }}\right| \leq 0.85$ for $q=1$. As in case (1), to get large Kerr parameters $\left|j_{\text {fin }}\right|>0.9$ in the absence of prolonged accretion we must start with large spins and avoid major mergers.

For each value of $q$, it is interesting to maximize and minimize the final spin resulting from a merger in the $\left(\left|j_{1}\right|,\left|j_{2}\right|\right)$ plane. The resulting extrema in the three different scenarios are plotted as functions of $q$ in Figure 3 . For each value of $q$ we average over the individual spin magnitudes $\left(\left(\left|\mathrm{j}_{1}\right|,\left|\mathrm{j}_{2}\right|\right)\right.$. Both $\left|\mathrm{j}_{1}\right|$ and $\left|\mathrm{j}_{2}\right|$ are assumed to be uniformly distributed between 0 and 1 . In the isotropic case, the allowed average values of the final spin range between the solid and dashed black lines. The most interesting feature is the appearance of a funnel at $q \gtrsim 0.1$ : major mergers tend to produce $\mathrm{BHs}$ with average spins very close to the value $\left|j_{\text {fin }}\right| \simeq 0.69$ resulting from equalmass, non-spinning mergers. For smaller $q$ the larger $\mathrm{BH}$ dominates the dynamics, and the final spin can be substantially larger or smaller than this value. Moving from isotropic to equatorial and aligned mergers can produce slightly larger maximum spins (as indicated by the solid lines of decreasing thickness), but in all three scenarios the most likely spin resulting from "major" mergers is very close to $\left|\mathrm{j}_{\text {fin }}\right| \simeq 0.69$. We now explore some consequences of these results for spin evolution in cosmological BH formation scenarios.

\section{COSMOLOGICAL SPIN EVOLUTION}

In Figure 4 we show histograms of the spin distribution of merging $\mathrm{BHs}$ in different redshift ranges. For simplicity, $\mathrm{BH}$ seeds at high redshift are assumed to be non-spinning. These plots are useful to isolate the contribution of mergers to the spin evolution of the whole $\mathrm{BH}$ population, and they are of direct interest for LISA observations of massive BH inspiral, merger and ringdown (Berti et al. 2005, 2006; Lang \& Hughes 2006; Kocsis et al. 2007). The three columns in each of the three plots correspond to the merging scenarios described above: isotropy, efficient alignment of the spins with L, and equatorial mergers.

In the left plot, spin evolution is due to mergers only and accretion is ignored. Consider first isotropic mergers (left column). At high $z$ most $\mathrm{BH}$ seeds have comparable masses, and since mergers with $q \gtrsim 0.1$ produce remnants with $\left|\mathrm{j}_{\text {fin }}\right| \sim 0.7$ (Figure 3) the spin distribution postmerger peaks around this value. Later on, small- $q$ mergers become more common, and on average (as shown in HB) they tend to spin down the hole, so the spin distribution flattens out. For aligned mergers (central column) the evolution is different. At high $z$, mergers of comparable-mass, non-spinning holes again produce a peak at $\left|j_{\text {fin }}\right| \sim 0.7$. However, if alignment is efficient mergers of spinning holes have a tendency to spin up the remnant, and eventually most BHs at small $z$ are rapidly spinning. In the equatorial merger scenario (right column) the less massive hole has marginal impact on the dynamics, and the overall spin distribution is qualitatively very similar to the case of alignment.

In the central plot spin evolution is due to both mergers and accretion, where accretion is modeled as in paper I: prograde or retrograde orbits are equally probable, and spins evolve according to Bardeen (1970). If enough mass is available to the hole, and the disc was initially counterrotating with respect to the hole, an overflip can eventually occur. In this scenario, accretion-induced spin-up is very efficient. For aligned (equatorial) mergers, $\gtrsim 90 \%$ of merging BHs at $z \lesssim 5(z \lesssim 2)$ have $j>0.9$. If mergers occur isotropically, on average they tend to spin down the holes, partially counteracting the accretion-induced spin-up. As a result, in this case the spin distribution post-merger has a long tail extending all the way down to $j \simeq 0$.

In the right plot we consider the chaotic accretion scenario. Now accretion happens in short-lived episodes, where the $\mathrm{BH}$ increases its mass by $0.1 \%$, and prograde or retrograde orbits are equally probable. Mergers per se are unlikely to produce fast-spinning remnants, and chaotic accretion is very efficient in spinning BHs down. 
Therefore, at all but the highest redshifts the spin distribution post-merger is roughly uniform in the range $j \in(0,0.7-0.8)$, and the chance of mergers involving high-spinning BHs is very low. LISA measurements of spins $j>0.9$ would strongly favor prolonged accretion and indicate that alignment is efficient in merger events. Conversely, measuring values of $j<0.9$ at $z \lesssim 5$ would indicate that accretion is chaotic (or negligible), or that alignment is not efficient.

For BH spin measurements based on X-ray spectroscopy (Miller 2007) and for the low-redshift observations of gravitational waves from compact objects falling into a massive $\mathrm{BH}$ (EMRIs), that could allow very accurate spin measurements (Barack \& Cutler 2004), we are interested in the spin distribution of the whole $\mathrm{BH}$ population. This distribution is shown in Figure 5.

When we consider the spin evolution under mergers only (left plot) the overall spin distribution is bimodal. One peak is around $j \sim 0$ because we assume that seed BHs are non-spinning, and some BHs never experience mergers. A second peak is located around $j \sim 0.7$ (for isotropic mergers) and it extends all the way up to $j \sim 1$ for aligned/equatorial mergers.

If accretion influences the evolution of BH spins, it dominates over mergers in determining the spin evolution of the whole BH population, confirming the results of Paper I. We stress that our accretion model is highly oversimplified, and does not take into account the different evolution of spins in different galactic environments: here we simply assume that accretion is triggered always and only by galaxy mergers. This is indeed a simplistic assumption, especially for low-redshift faint AGNs: see Volonteri et al. (2007) for a detailed analysis of the connection between spin evolution and galaxy morphology.

Whatever the dominant merger scenario, under prolonged accretion (central plot) spin-up is very efficient, and a large fraction of all BHs has spin $j>0.9$. On average isotropic mergers tend to spin BHs down: if isotropy is the norm, $\sim 30 \%$ of the whole BH population has $j<0.9$, with a roughly uniform distribution. For aligned and equatorial mergers spin-down is less efficient, and only $\sim 10 \%$ of all BHs have $j<0.9$.

The chaotic accretion scenario (right plot) predicts a completely different distribution. In this case most BHs ( $\sim 50-80 \%$ of the total) have $j \lesssim 0.1$. When the average spins are so low mergers act to spin BHs up, and the spinup is more efficient in the aligned and equatorial cases. This produces a roughly uniform spin distribution in the range $j \in(0.1,0.7)$. Interestingly, the tail of the distribution never extends above $j \sim 0.8$, even for small redshifts. The measurement of large BH spins, such as the value of $j \sim 0.99$ claimed by Brenneman \& Reynolds (2006) for MCG-06-30-15 (see also Elvis et al. 2002; Wang et al. 2006) would presumably indicate that chaotic accretion is not the norm. In any case, $\mathrm{BH}$ spin measurements should easily distinguish between chaotic and prolonged accretion. Both for merging $\mathrm{BHs}$ and for the whole $\mathrm{BH}$ population, under chaotic accretion maximal Kerr BHs are extremely unlikely, and the distribution peaks at $j<0.7$ for all $z<10$.

Suppose that LISA measurements of the spin resulting from a binary merger yield $j>0.9$. Then it is hard to tell if spin is accretion-independent, but alignment is efficient (left plot in Figure 4) or if instead prolonged accretion is responsible for spinning BHs up (central plot). Luckily, this degeneracy is broken when we consider the whole population. If accretion is negligible and mergers are not isotropic, only $\lesssim 50 \%$ of all BHs have spins $j>0.9$. If instead spin-up is due to accretion, the population is largely dominated by spins $j \sim 1$ (compare the left and central plots in Figure 5). EMRIs (Barack \& Cutler 2004) and X-ray observations of the $\mathrm{Fe} \mathrm{K} \alpha$ line by planned missions such as Constellation-X (Miller 2007) will probe the whole population at low redshifts $(z<2)$, while observations of the inspiral (Lang \& Hughes 2006) and ringdown (Berti et al. 2006) of massive BHs can provide a census of $\mathrm{BH}$ spins over a wider range of epochs. A combination of these techniques (and possibly others) has the potential to probe unequivocally the physical processes involved in the evolution of $\mathrm{BH}$ spins.

\section{CONCLUSIONS}

Understanding how fast BHs spin is as important as understanding how they grow in mass. The spin and mass evolution of BHs are intimately linked. The expected spin of a hole depends on whether it gained most of its mass via mergers or accretion. Conversely, the spin influences how efficiently BHs accrete mass, determining the massto-energy conversion efficiency in radiatively efficient accretion phases. The spin also determines how much energy can be extracted from aBH. Assuming that relativistic jets are powered by rotating BHs through the BlandfordZnajek mechanism, the so-called "spin paradigm" asserts that powerful relativistic jets are produced in AGN with fast rotating $\mathrm{BHs}$ (Blandford 1990).

Studying the role of $\mathrm{BH}$ mergers in the evolution of $\mathrm{BH}$ spins has been daunting until recently. Due to remarkable advances in numerical relativity, we finally have a quantitative understanding of the spins resulting from comparable-mass binary $\mathrm{BH}$ mergers (see e.g. Pretorius 2007). Here we use numerical relativity results to investigate plausible scenarios of spin evolution through mergers and accretion in a cosmological context. The co-evolution of $\mathrm{BH}$ masses and spins is studied self-consistently: mergers and accretion determine $\mathrm{BH}$ spins, which in turn lend to the calculation of the radiative efficiency during accretion episodes.

We focus on three scenarios for the mass and spin coevolution: (1) spins evolve only through mergers, (2) spins evolve through mergers and prolonged accretion episodes, (3) spins evolve through mergers and short-lived (chaotic) accretion episodes. If $\mathrm{BHs}$ accreted most of their mass through prolonged disc-mode accretion, by adding material with constant angular momentum axis, they would end up spinning rapidly. If instead BHs built-up their mass via short-lived episodes with uncorrelated angular momentum axis, the typical spin of BHs would be very low.

We further consider how the dynamics of $\mathrm{BH}$ mergers influences the final spin. The mutual directions of the spins of the holes in a binary with respect to the orbital plane strongly affect the final spin of the remnant. If the spins align efficiently with the orbital angular momentum, the spin of the merger remnant is larger than in the case of random orientations (see Figures 2 and 3). We consider 
three physically motivated cases: (1) random orientations (isotropy), which is expected if $\mathrm{BH}$ binaries are in gaspoor host galaxies, or if the orbital plane is not aligned with the disc plane; (2) alignment between spins and orbital angular momentum, which Bogdanović et al. (2007) suggest should be the norm for binaries forming during gas-rich galaxy mergers; (3) equatorial orbits, where the smaller BH inspirals in the equatorial plane of the larger. Equatorial mergers could occur if dynamical friction brings the smaller hole into the plane of a central disc before it gets into the relativistic regime.

Except in the case of aligned mergers, we find that a sequence of BH mergers can lead to large spins $\left|\mathrm{j}_{\text {fin }}\right|>0.9$ only if $\mathrm{BHs}$ start already with large spins and they do not experience many major mergers. This is illustrated in Figure 3. where we show that (on average) isotropic mergers with $q>0.05$ lead to $\left|\mathrm{j}_{\text {fin }}\right|<0.9$. Therefore, the common assumption that mergers between BHs of similar mass always lead to large spins (e.g., Wilson \& Colbert 1995) needs to be revised. In the isotropic case, numerical relativity results imply that major mergers tend to produce BHs with average spins very close to the value $\left|\mathrm{j}_{\text {fin }}\right| \simeq 0.69$ resulting from equal-mass, non-spinning mergers (see again Figure 3).

Our models can be used to test the discriminating power of direct observational techniques: gravitationalwave measurements and X-ray spectroscopy. LISA measurements can provide information on the spins of the two
BHs in a binary prior to merger (inspiral) and on the spin of the merger remnant (ringdown). Such measurements can unequivocally inform us on the typical spin of the $\mathrm{BH}$ population (Figure 4). However, if LISA measurements of the spin resulting from a binary merger yield $j>0.9$ a degeneracy remains: a distribution skewed towards large values can occur if spin is accretion-independent, but alignment is efficient (left plot in Figure 4), or if instead prolonged accretion is responsible for spin up (central plot).

This degeneracy is broken by coupling spin measurements of binaries to either X-ray spectroscopy or EMRIs, as these techniques sample the whole population. If ironline measurements and gravitational-wave observations of extreme mass-ratio inspirals (EMRIs) only yield dimensionless spins $j=J / M^{2}>0.9$, then prolonged accretion should be responsible for spin-up, and chaotic accretion scenarios would be very unlikely. If instead only a fraction of the whole population of low-redshift BHs spins rapidly, spin-alignment during binary mergers (rather than prolonged accretion) could be responsible for spin-ups.

We thank Curt Cutler, Tom Prince and the LISA science performance evaluation taskforce for discussions. E.B.'s research was supported by an appointment to the NASA Postdoctoral Program at JPL, administered by Oak Ridge Associated Universities through a contract with NASA. Copyright 2008 California Institute of Technology. Government sponsorship acknowledged.

\section{REFERENCES}

Arnaud, K. A. et al. 2007, Classical and Quantum Gravity, 24, 551

Baker, J. G. et al. 2007, ApJ, 668, 1140

Barack, L. \& Cutler, C. 2004, Phys. Rev. D, 69, 082005

Bardeen, J. M. 1970, Nature, 226, 64

Berti, E. 2006, Classical and Quantum Gravity, 23, 785

Berti, E., Buonanno, A., \& Will, C. M. 2005, Phys. Rev. D, 71, 084025

Berti, E., Cardoso, V., \& Will, C. M. 2006, Phys. Rev. D, 73, 064030

Berti, E. et al. 2007a, Phys. Rev. D, 76, 064034

- 2007b, arXiv:0711.1097

Blandford, R. D. 1990, Active Galactic Nuclei, 161

Bogdanović, T., Reynolds, C. S., \& Miller, M. C. 2007, ApJ, 661, L147

Boyle, L., Kesden, M., \& Nissanke, S. 2007, arXiv:0709.0299

Brenneman, L. W. \& Reynolds, C. S. 2006, ApJ, 652, 1028

Buonanno, A., Kidder, L. E., \& Lehner, L. 2007, arXiv:0709.3839

Campanelli, M., Lousto, C., Zlochower, Y., \& Merritt, D. 2007, ApJ, $659, \mathrm{~L} 5$

Campanelli, M., Lousto, C. O., \& Zlochower, Y. 2006, Phys. Rev. D, 74,041501

Elvis, M., Risaliti, G., \& Zamorani, G. 2002, ApJ, 565, L75

Gammie, C. F., Shapiro, S. L., \& McKinney, J. C. 2004, ApJ, 602, 312

Herrmann, F. et al. 2007, Phys. Rev. D, 76, 084032

Hughes, S. A. \& Blandford, R. D. 2003, ApJ, 585, L101

King, A. R. et al. 2005, MNRAS, 363, 49

King, A. R. \& Pringle, J. E. 2006, MNRAS, 373, L90
King, A. R., Pringle, J. E., \& Hofmann, J. A. 2008, MNRAS, 229

Kocsis, B., Haiman, Z., \& Menou, K. 2007, arXiv:0712.1144

Lang, R. N. \& Hughes, S. A. 2006, Phys. Rev. D, 74, 122001

Lodato, G. \& Pringle, J. E. 2006, MNRAS, 368, 1196

Marronetti, P. et al. 2007, arXiv:0709.2160

Merritt, D., \& Ekers, R. D. 2002, Science, 297, 1310

Miller, J. M. 2007, ARA\&A, 45, 441

Narayan, R. 2005, New Journal of Physics, 7, 199

Pollney, D. et al. 2007, Phys. Rev. D, 76, 124002

Pretorius, F. 2007, arXiv:0710.1338

Rezzolla, L. et al. 2007a, arXiv:0712.3541

- 2007b, arXiv:0708.3999

-. 2007c, arXiv:0710.3345

Schnittman, J. D. 2004, Phys. Rev. D, 70, 124020

Sesana, A., Volonteri, M., \& Haardt, F. 2007, MNRAS, 377, 1711

Shapiro, S. L. 2005, ApJ, 620, 59

Sperhake, U. et al. 2007, arXiv:0710.3823

Thorne, K. S. 1974, ApJ, 191, 507

Tichy, W. \& Marronetti, P. 2007, Phys. Rev. D, 76, 061502

Volonteri, M., Haardt, F., \& Madau, P. 2003, ApJ, 582, 559

Volonteri, M., Madau, P., Quataert, E., \& Rees, M. J. 2005, ApJ, 620,69

Volonteri, M., Sikora, M., \& Lasota, J.-P. 2007, ApJ, 667, 704

Wang, J.-M., Chen, Y.-M., Ho, L. C., \& McLure, R. J. 2006, ApJ, 642, L111

Wilson, A. S., \& Colbert, E. J. M. 1995, ApJ, 438, 62 

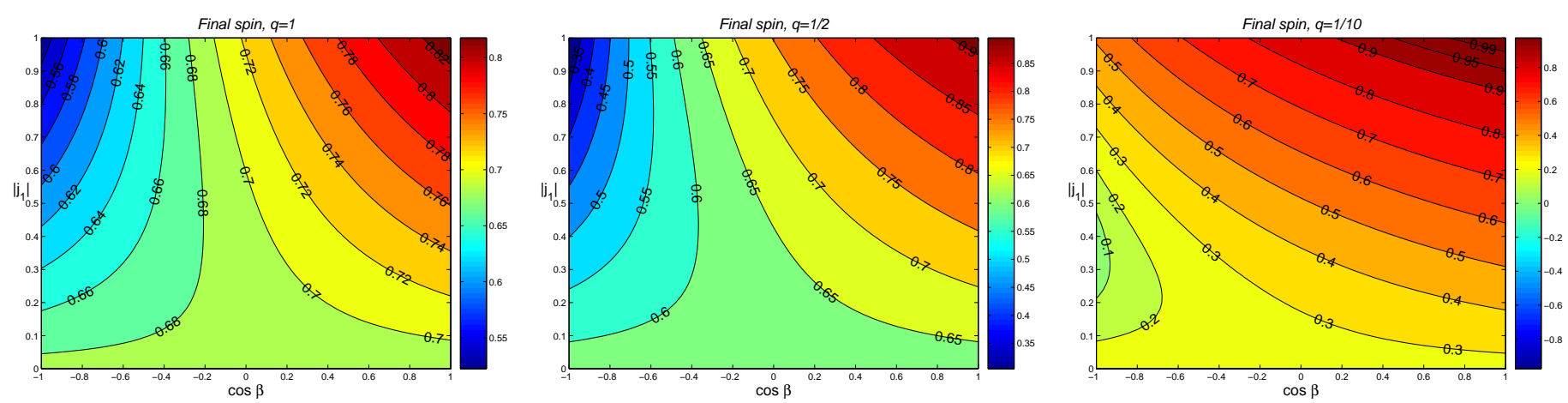

Fig. 1. - Update of Figure 1 in Hughes \& Blandford (2003). Here we assume $\left|\mathrm{j}_{2}\right|=0$ (so that $\cos \alpha$ and $\cos \gamma$ are irrelevant) and we show the influence of the orbital inclination $\cos \beta$ on the final spin for different values of $\left|j_{1}\right|$.
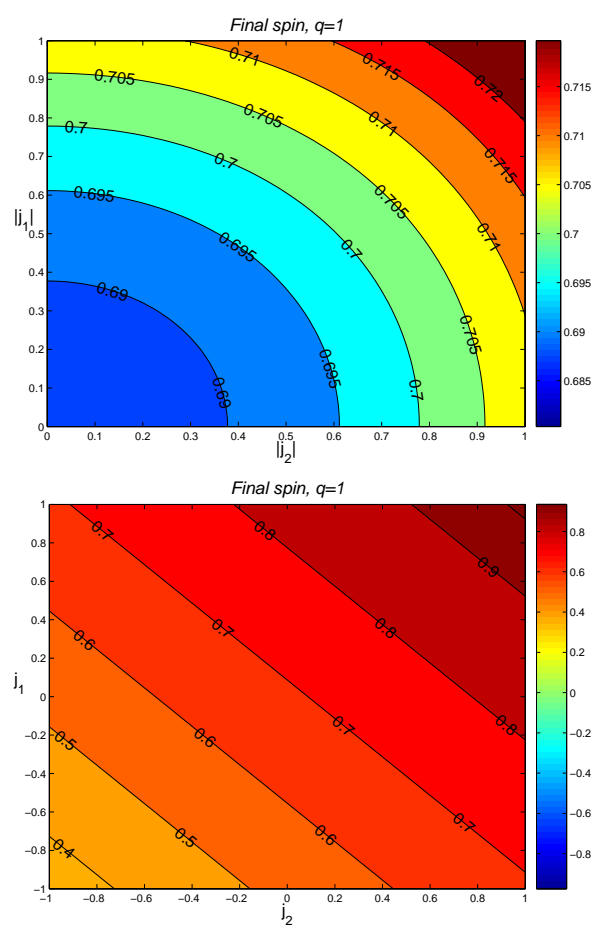

Final spin, $q=1$

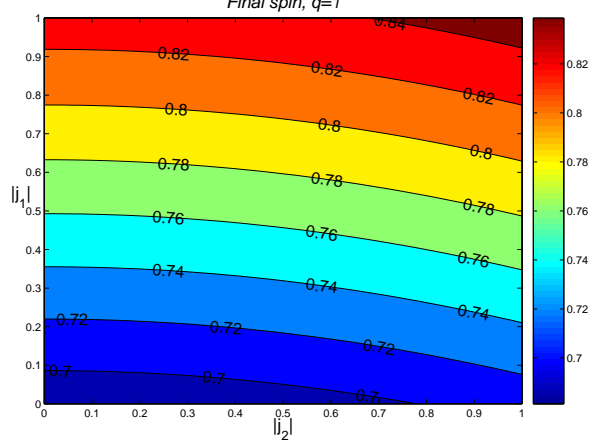

Final spin, $q=1 / 2$

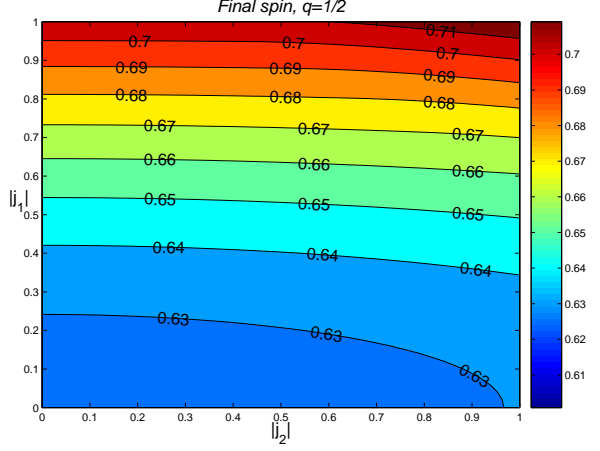

Final spin, $q=1 / 2$

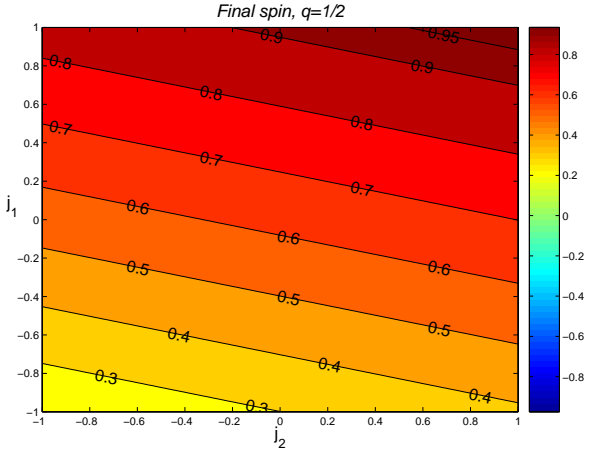

Final spin, $q=1 / 2$

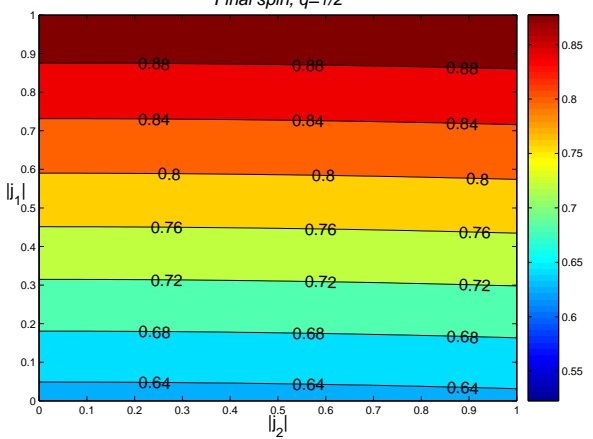

Final spin, $q=1 / 10$
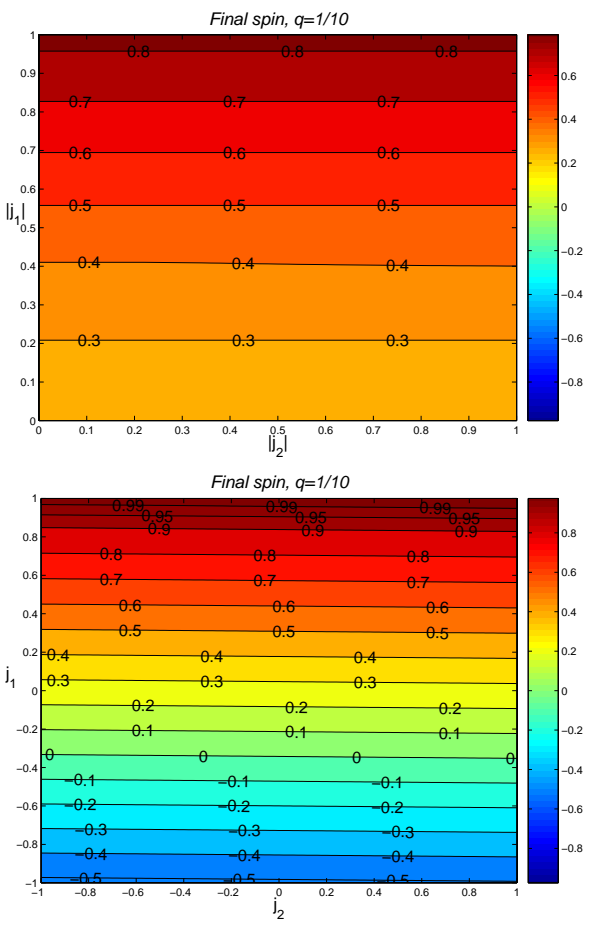

Final spin, $q=1 / 10$

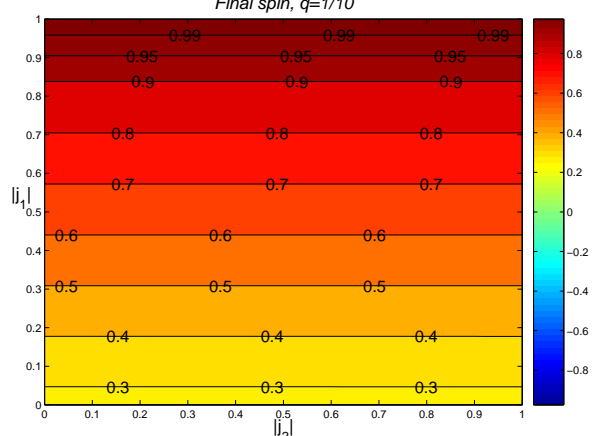

FIG. 2.- Contour plots of the final spin for different mass ratios (left to right: $q=1, q=1 / 2$ and $q=1 / 10$, respectively) in the $\left(\left|j_{1}\right|\right.$, $\left|\mathrm{j}_{2}\right|$ ) plane. Recall that $M_{2} \leq M_{1}$. We consider three different scenarios. In the top row we average over the sky assuming isotropy on all three angles. In the middle row we assume that alignment is efficient, as proposed by Bogdanović et al. (2007). By convention, in this case $j_{i}<0$ means that the spin of $\mathrm{BH} i$ is antialigned (rather than aligned) with the orbital angular momentum. In the bottom row we set cos $\beta=1$ (so the spin of more massive BH is aligned with the orbital angular momentum, and the smaller hole orbits in the equatorial plane of the larger) and we assume isotropy in $(\cos \alpha, \cos \gamma)$. 


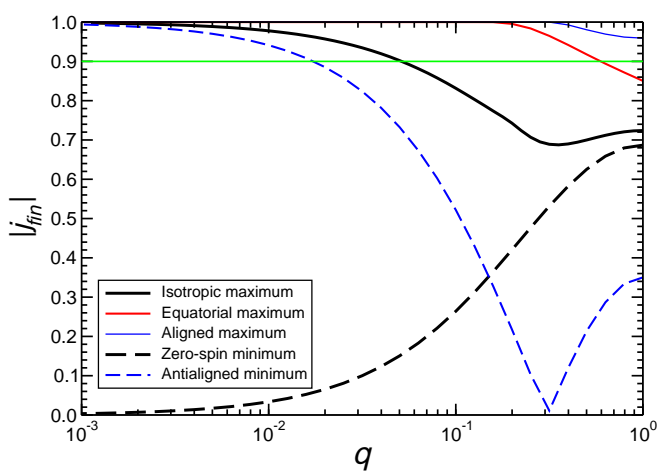

FIG. 3. - Solid black (thick) line: maximum average spin in the isotropic case. Solid red (medium thickness) line: maximum average spin for equatorial inspirals. Solid blue (thin) line: maximum spin in the aligned case. Dashed black (thick) line: minimum average spin in the isotropic case. Since the minimum is attained when $\left|j_{1}\right|=\left|j_{2}\right|=0$, this line is also the minimum average spin attainable by equatorial inspirals, or the minimum spin attainable in the "aligned" case when we rule out the possibility of antialignment. Dashed blue (thin) line: if we do allow for antialignment, the minimum spin can become negative (we have a spin flip) when $q \lesssim 0.3$ or so. The horizontal (green) line corresponds to a final spin $\left|j_{\text {fin }}\right|=0.9$. When $q$ is close to one, such large spins are only achievable if alignment is efficient.
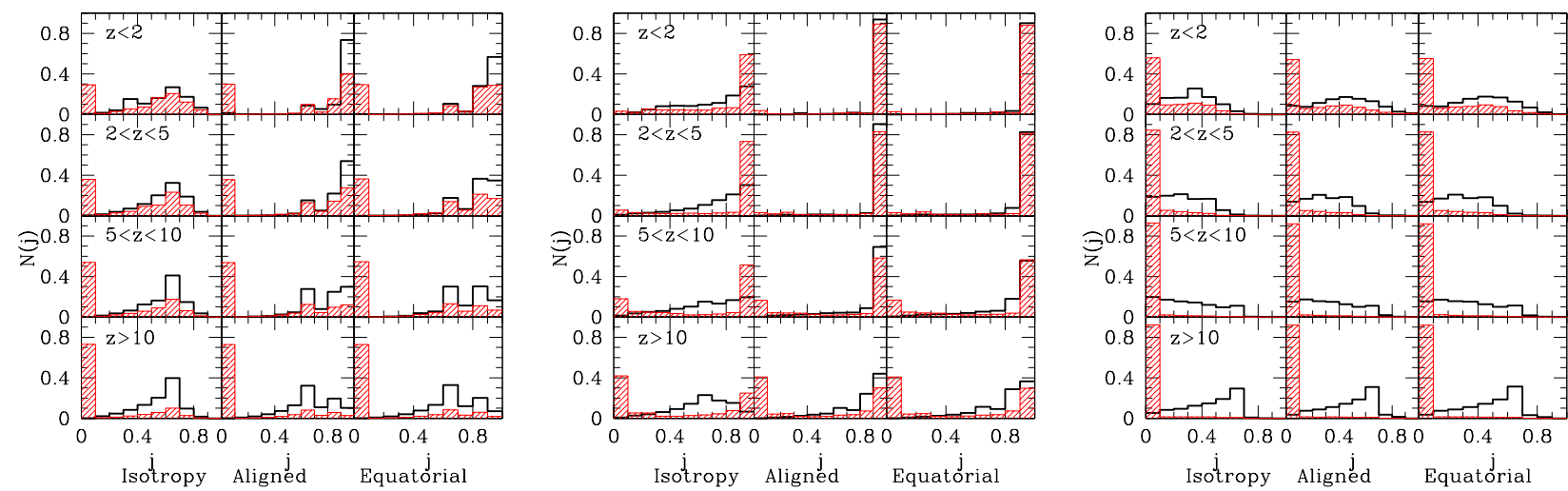

FIG. 4.- Spin evolution of merging BHs due to: mergers only (left); mergers and prolonged accretion (center); mergers and chaotic accretion (right). In each plot we consider three representative merger scenarios (see text) and we show histograms of the spin distribution for different ranges of variability of the redshift $z$. Hatched (red) histogram: spins of the binary members before merger. Thick (black) histogram: spins of BHs after merger.
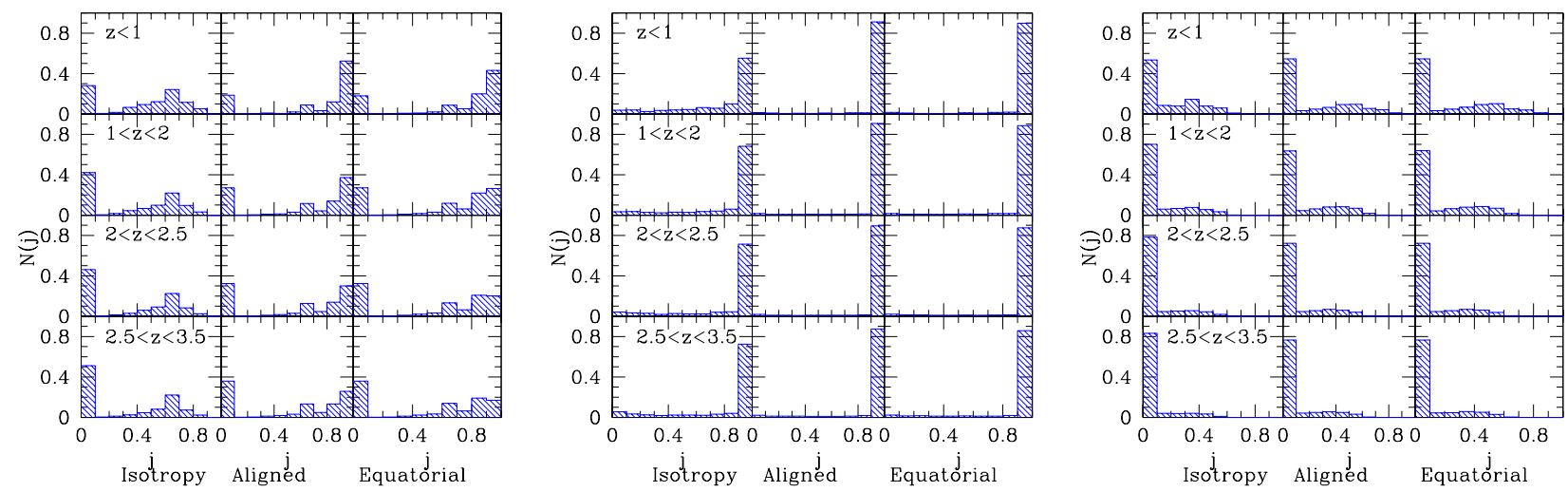

FIG. 5.- Spin evolution of all BHs due to mergers only (left), mergers plus standard accretion (center) and mergers plus chaotic accretion (right). 